\title{
Sleep apnoea, insulin resistance and diabetes: the first step is in the fat
}

\author{
Isaac Almendros ${ }^{1,2}$ and Francisco García-Río $1,3,4$
}

Affiliations: ${ }^{1}$ Centro de Investigación Biomédica en Red de Enfermedades Respiratorias (CIBERES), Madrid, Spain. ${ }^{2}$ Unitat de Biofísica i Bioenginyeria, Facultad de Medicina, Universitat de Barcelona-IDIBAPS, Barcelona, Spain. ${ }^{3}$ Servicio de Neumología, Hospital Universitario La Paz, IdiPAZ, Madrid, Spain. ${ }^{4}$ Departamento de Medicina, Universidad Autónoma de Madrid, Madrid, Spain.

Correspondence: Francisco García-Río, Servicio de Neumología, Hospital Universitario La Paz, Paseo de la Castellana, 261, 28046 Madrid, Spain. E-mail: fgr01magmail.com

@ERSpublications

Intermittent hypoxia's effect on adipose tissue induces insulin resistance: a first step in the OSA-diabetes link http://ow.ly/UzIm309kEMi

Cite this article as: Almendros I, García-Río F. Sleep apnoea, insulin resistance and diabetes: the first step is in the fat. Eur Respir J 2017; 49: 1700179 [https://doi.org/10.1183/13993003.00179-2017].

Over the last few years, the relationship between obstructive sleep apnoea (OSA) and various metabolic disorders, especially type 2 diabetes (T2D), has emerged strongly. This circumstance is particularly relevant in terms of public healthcare, considering that 415 million people were affected by diabetes in 2015, with an expected prevalence of 642 million by 2040. To this, we must also add another 318 million individuals with features indicating future risk for developing T2D, including fasting hyperglycaemia, impaired glucose tolerance and insulin resistance [1]. The healthcare expenditure for diabetes in Europe was about $€ 75$ billion in 2011 , which is projected to increase to $€ 90$ billion by $2030[1,2]$.

The development of T2D is the consequence of a series of concatenated alterations. In healthy individuals, insulin secretion by the pancreatic $\beta$-cells allows for normal glucose disposal in insulin-sensitive tissues, mainly adipose tissue, the liver and muscles. In predisposed individuals, there is a reduced response to insulin-stimulated glucose uptake in adipose tissue and the liver, known as insulin resistance, which is compensated by a rise in insulin production [3]. However, the compensatory capacity of the increased peripheral insulin demand is lost when the insulin-secretory capacity of the pancreatic $\beta$-cells is compromised. In a first phase, insulin resistance and impaired insulin secretion only cause postprandial hyperglycaemia, a characteristic of impaired glucose tolerance. In more advanced phases with a more pronounced deterioration of insulin production, persistent hyperglycaemia originates in the fasting state, corresponding with T2D [4].

To date, there is much clinical-epidemiological evidence to support the relationship of OSA with both insulin resistance and T2D [5]. Overall, it has been reported that the prevalence of prediabetes, assessed by insulin resistance and glucose intolerance, is higher in OSA patients than in controls, with estimates varying from 20 to $67 \%$ [6]. Several cross-sectional studies have shown that OSA impairs insulin sensitivity and glucose tolerance $[7,8]$, and it has been demonstrated that OSA is independently related to the development of insulin resistance, while the oxygen desaturation index is the main determining factor [9]. This latter finding, which has been corroborated by other studies [10], is particularly important, as it involves the hypoxic stress of OSA in the development of insulin resistance. Finally, OSA has also been independently associated with T2D [11]. In fact, in a very large nationwide study of a health insurance claims database, T2D was significantly more prevalent in OSA patients than in matched controls from all age groups [12]. In the same vein, in a cross-sectional analysis of the European Sleep Apnoea Cohort

Received: Jan 242017 | Accepted after revision: Feb 042017

Conflict of interest: None declared.

Copyright CERS 2017 
study, T2D prevalence increased with OSA severity from $6.6 \%$ in subjects without OSA to $28.9 \%$ in those with severe OSA, and OSA severity was associated with glycaemic control in patients with T2D [13].

In order to consolidate the relationships between OSA, insulin resistance and diabetes, it is necessary to identify pathogenic pathways connecting the two key elements of OSA - intermittent hypoxia (IH) and sleep fragmentation - with loss of sensitivity to insulin in its target tissues and pancreatic islet $\beta$-cell dysfunction. In addition to providing biological plausibility to the OSA-T2D relationship, the characterisation of pathogenic pathways could provide for the identification of targets on which to act or the definition of patient profiles for those at risk or susceptible to intervention. Thus far, potential mechanisms have been proposed that could explain the association between OSA and metabolic dysfunction, including activation of the sympathetic nervous system, changes in hypothalamic-pituitaryadrenal axis activity, formation of reactive oxygen species, and increases in inflammatory cytokines and adipocyte-derived factors, such as adiponectin, leptin and resistin $[2,14]$.

Of all the possible pathways involved, numerous studies have investigated the relationship between $\mathrm{IH}$ and insulin resistance, assessing the effect of hypoxia-reoxygenation cycles on insulin target tissues. In several rodent models, it has been reported that chronic exposure to $\mathrm{IH}$ induces insulin resistance [15] and impairs glucose tolerance [16]. It has also been shown that healthy subjects who have undergone acute episodes of $\mathrm{IH}$ experience a decrease in insulin sensitivity [17]. However, although IH appears to be responsible for insulin resistance, the mechanisms involved remain unclear. The article in this issue of the European Respiratory Journal by MURPHY et al. [18] provides important novel data demonstrating that IH decreases insulin sensitivity in lean and obese mice as well as cultured adipocytes, impeding insulin-mediated glucose uptake and, subsequently, inducing insulin resistance. In both murine epididymal visceral fat and adipocytes, IH down-regulates insulin-receptor substrate 1 (IRS-1) mRNA, decreasing insulin-induced tyrosine phosphorylation of the insulin receptor (IR $\beta$ ) and IRS, as well as phosphorylation of Akt in adipocytes. Thus, IH leads to decreased functionality of adipose tissue, with inhibition of the insulin signalling pathway and down-regulation of IRS-1 mRNA. In addition, interesting information is also provided about the impact of IH on the adipose inflammatory phenotype. In studies in murine and in vitro models, it was observed that $\mathrm{IH}$ induces a pro-inflammatory phenotype of visceral adipose tissue with pro-inflammatory M1 adipose-tissue macrophage polarisation correlating with the degree of insulin resistance. These results are also in accordance with a very recent approach carried out by GozAL and co-workers $[19,20]$. In the absence of a definitive mechanistic connection between M1 inflammation of adipose tissue and IRS down-regulation, the data generated provide new insights into understanding the effect of IH on one of the main target tissues of insulin.

In line with the M1 polarisation identified in adipose tissue, MURPHY et al. [18] also describe that, in subjects without comorbidities, OSA severity correlates with serum levels of CD163, a surface marker characteristic of M2 macrophages, but whose soluble fraction requires M1-dependent cleavage [21]. It has therefore been considered a pro-inflammatory biomarker of several cardiovascular diseases, including insulin resistance and T2D [22]. Along the same lines, the demonstration of systemic M1 polarisation in patients with OSA related to nocturnal hypoxia intensity could contrast with the previous description of M2 polarisation in animal models with tumour infiltration subjected to intermittent hypoxia [23]. However, it should be kept in mind that this change in IH-induced macrophage polarisation could represent a merely local effect, relative to the proximity of tumour cells, because it has been described that while macrophages surrounding the tumour and those from the ipsilateral adipose tissue exhibit an M2 phenotype, an infiltration of preferentially M1 macrophages is observed in the contralateral adipose tissue [24]. Nonetheless, current knowledge of $\mathrm{IH}$-induced macrophage polarisation does not allow us to conclude whether the two patterns identified are attributable solely to a local effect or whether there could be contributing systemic factors leading to different risk patterns in the development of comorbidity: metabolic-vascular (M1) versus pro-tumour (M2).

Identification of the effect of hypoxia on the inflammatory response of adipose tissue, as well as a better definition of the pathways involved in the reduced insulin sensitivity of this insulin target organ, will enable us to complete part of the sequence of glucose metabolism alterations induced by OSA, which will necessarily involve more organs and mechanisms (figure 1). In particular, some information is available on the effect of sleep fragmentation (SF). In a similar manner to $\mathrm{IH}$, a shift towards the M1 phenotype in macrophages of mice subjected to chronic SF has been reported [25]. In addition, some evidence seems to indicate that it could intervene in the metabolic dysfunction observed in OSA. Thus, it has been demonstrated that shorter sleep duration negatively affects glucose metabolism [26], and, in a recent meta-analysis, the relative risk for the development of T2D associated with short sleep was estimated at 1.28 [27]. Although specific information on the metabolic consequences of sleep fragmentation is still limited, two independent groups have also shown negative effects of sleep fragmentation on insulin sensitivity in healthy participants $[28,29]$. In addition, prospective population-based studies have shown an association between self-reported poor sleep quality and the incidence of T2D [30]. 


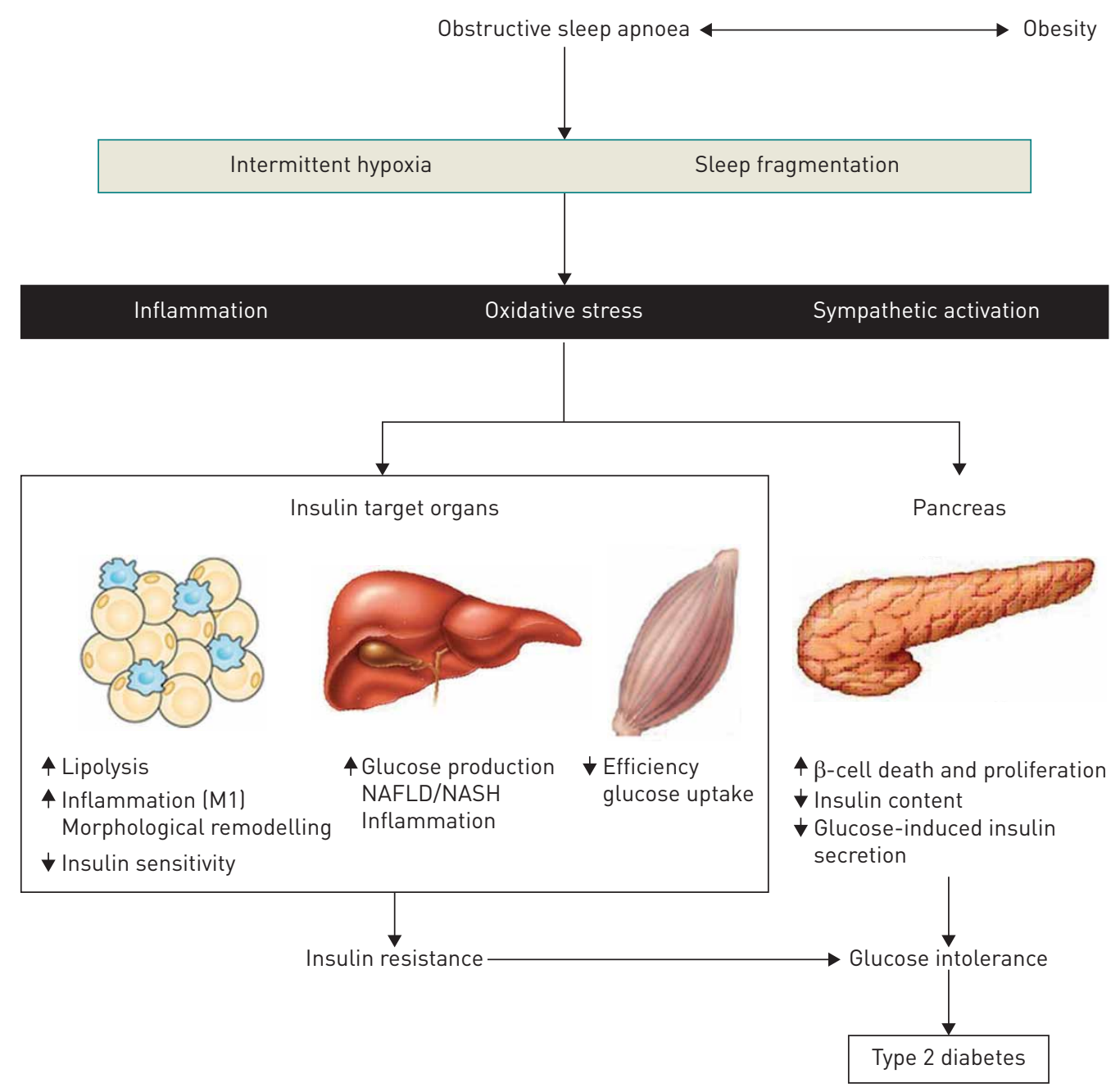

FIGURE 1 Potential mechanisms linking obstructive sleep apnoea to alteration in glucose metabolism. M1: pro-inflammatory macrophages; NAFLD: non-alcoholic fatty liver disease; NASH: non-alcoholic steatohepatitis.

Another important aspect pending clarification lies in the identification of OSA factors that contribute to the dysfunction of pancreatic $\beta$-cells and justify the transition from pre-diabetes (insulin resistance) to T2D [31]. Although still preliminary, some data have suggested the involvement of inflammation, oxidative stress and increased sympathetic tone induced by OSA [2,3]. Finally, in order to establish a partnership between OSA and T2D, it is necessary to determine the effect of apnoea-hypopnoea suppression on insulin resistance and glycaemic control in patients with OSA and T2D, the latter of which is still the subject of considerable debate $[32,33]$.

In short, the relationship between OSA and diabetes, which is supported by considerable epidemiological evidence, will be reinforced by the precise characterisation of its pathogenic mechanisms. Identification of the routes of action of $\mathrm{IH}$ on adipose tissue to induce insulin resistance is a first step in this regard.

\section{References}

1 International Diabetes Federation. Global Burden: Prevalence and Projections, 2015 and 2040. Seventh Edition. Brussels, IDF, 2015. www.diabetesatlas.org/resources/2015-atlas.html Date last accessed: March 4, 2017.

2 Aurora RN, Punjabi NM. Obstructive sleep apnoea and type 2 diabetes mellitus: a bidirectional association. Lancet Respir Med 2013; 1: 329-338.

3 Donath MY. Targeting inflammation in the treatment of type 2 diabetes: time to start. Nat Rev Drug Discov 2014; 13: 465-476.

4 Mari A, Tura A, Natali A, et al. Impaired beta cell glucose sensitivity rather than inadequate compensation for insulin resistance is the dominant defect in glucose intolerance. Diabetologia 2010; 53: 749-756.

5 Martínez Cerón E, Casitas Mateos R, García-Río F. Sleep apnea-hypopnea syndrome and type 2 diabetes. A reciprocal relationship? Arch Bronconeumol 2015; 51: 128-139.

$6 \quad$ Pamidi S, Tasali E. Obstructive sleep apnea and type 2 diabetes: is there a link? Front Neurol 2012; 3: 126.

7 Ip MS, Lam B, Ng MM, et al. Obstructive sleep apnea is independently associated with insulin resistance. Am J Respir Crit Care Med 2002; 165: 670-676. 
8 Punjabi NM, Shahar E, Redline S, et al. Sleep disordered breathing, glucose intolerance, and insulin resistance: the Sleep Heart Health Study. Am J Epidemiol 2004; 160: 521-530.

9 Lindberg E, Theorell-Haglöw J, Svensson M, et al. Sleep apnea and glucose metabolism: a long-term follow-up in a community-based sample. Chest 2012; 142: 935-942.

10 Polotsky VY, Patil SP, Savransky V, et al. Obstructive sleep apnea, insulin resistance, and steatohepatitis in severe obesity. Am J Respir Crit Care Med 2009; 179: 228-234.

11 Kendzerska T, Gershon AS, Hawker G, et al. Obstructive sleep apnea and incident diabetes. A historical cohort study. Am J Respir Crit Care Med 2014; 190: 218-225.

12 Mokhlesi B, Ham SA, Gozal D. The effect of sex and age on the comorbidity burden of OSA: an observational analysis from a large nationwide US health claims database. Eur Respir J 2016; 47: 1162-1169.

13 Kent BD, Grote L, Ryan S, et al. Diabetes mellitus prevalence and control in sleep-disordered breathing: the European Sleep Apnea Cohort (ESADA) study. Chest 2014; 146: 982-990.

14 Martínez-Ceron E, Fernández-Navarro I, Garcia-Rio F. Effects of continuous positive airway pressure treatment on glucose metabolism in patients with obstructive sleep apnea. Sleep Med Rev 2016; 25: 121-130.

15 Iiyori $\mathrm{N}$, Alonso LC, $\mathrm{Li} \mathrm{J}$, et al. Intermittent hypoxia causes insulin resistance in lean mice independent of autonomic activity. Am J Respir Crit Care Med 2007; 175: 851-857.

16 Polak J, Shimoda LA, Drager LF, et al. Intermittent hypoxia impairs glucose homeostasis in C57BL6/J mice: partial improvement with cessation of the exposure. Sleep 2013; 36: 1483-1490.

17 Louis M, Punjabi NM. Effects of acute intermittent hypoxia on glucose metabolism in awake healthy volunteers. J Appl Physiol 2009; 106: 1538-1544.

18 Murphy AM, Thomas A, Crinion SJ, et al. Intermittent hypoxia in obstructive sleep apnoea mediates insulin resistance through adipose tissue inflammation. Eur Respir J 2017; 49: 1601731.

19 Gozal D, Gileles-Hillel A, Cortese R, et al. Visceral white adipose tissue following chronic intermittent and sustained hypoxia in mice. Am J Respir Cell Mol Biol 2017; in press [https://doi.org/10.1165/rcmb.2016-0243OC].

20 Carreras A, Zhang SX, Almendros I, et al. Resveratrol attenuates intermittent hypoxia-induced macrophage migration to visceral white adipose tissue and insulin resistance in male mice. Endocrinology 2015; 156: 437-443.

21 Etzerodt A, Maniecki MB, Moller $\mathrm{K}$, et al. Tumour necrosis factor $\alpha$-converting enzyme (TACE/ADAM 17 ) mediates ectodomain shedding of the scavenger receptor CD163. J Leukoc Biol 2010; 88: 1201-1205.

22 Urbonaviciene G, Martin-Ventura JL, Lindholt IS, et al. Impact of soluble TWEAK and CD163/TWEAK ratio on longterm cardiovascular mortality in patients with peripheral arterial disease. Atherosclerosis 2011; 219: 892-899.

23 Almendros I, Wang Y, Becker L, et al. Intermittent hypoxia-induced changes in tumour-associated macrophages and tumour malignancy in a mouse model of sleep apnea. Am J Respir Crit Care Med 2014; 189: 593-601.

24 Almendros I, Gileles-Hillel A, Khalyfa A, et al. Adipose tissue macrophage polarization by intermittent hypoxia in a mouse model of OSA: effect of tumour microenvironment. Cancer Lett 2015; 361: 233-239.

25 Carreras A, Zhang SX, Peris E, et al. Effect of resveratrol on visceral white adipose tissue inflammation and insulin sensitivity in a mouse model of sleep apnea. Int J Obes (Lond) 2015; 39: 418-423.

26 Gottlieb DJ, Punjabi NM, Newman AB, et al. Association of sleep time with diabetes mellitus and impaired glucose tolerance. Arch Intern Med 2005; 165: 863-867.

27 Cappuccio FP, D'Elia L, Strazzullo P, et al. Quantity and quality of sleep and incidence of type 2 diabetes: a systematic review and meta-analysis. Diabetes Care 2010; 33: 414-420.

28 Stamatakis KA, Punjabi NM. Effects of sleep fragmentation on glucose metabolism in normal subjects. Chest 2010; 137: 95-101.

29 Tasali E, Leproult R, Ehrmann DA, et al. Slow-wave sleep and the risk of type 2 diabetes in humans. Proc Natl Acad Sci USA 2008; 105: 1044-1049.

30 Meisinger C, Heier M, Loewel $\mathrm{H}$. Sleep disturbance as a predictor of type 2 diabetes mellitus in men and women from the general population. Diabetologia 2005; 48: 235-241.

31 American Diabetes Association. Diagnosis and classification of diabetes mellitus. Diabetes Care 2010; 33: Suppl. 1, S62-S69.

32 Martínez-Cerón E, Barquiel B, Bezos AM, et al. Effect of continuous positive airway pressure on glycemic control in patients with obstructive sleep apnea and type 2 diabetes. A randomized clinical trial. Am J Respir Crit Care Med 2016; 194: 476-485.

33 Shaw JE, Punjabi NM, Naughton MT, et al. The effect of treatment of obstructive sleep apnea on glycemic control in type 2 diabetes. Am J Respir Crit Care Med 2016; 194: 486-492. 